\title{
Pairing of integrins with ECM proteins determines migrasome formation
}

Cell Research (2017) 27:1397-1400. doi:10.1038/cr.2017.108; published online 22 August 2017

\section{Dear Editor,}

Recently we reported the discovery of migrasome, a new organelle of migrating cells [1]. Migrasomes are large vesicles that grow on the tips or intersections of retraction fibers at the rear of migrating cells. Following cell migration, the retraction fibers eventually break and the migrasomes become detached. The migrasomes and their contents, including cytosolic components and vesicles of unknown origin, are released into the extracellular space — a process we named as migracytosis. We speculated that migracytosis may play important roles in cell-cell communication.

Integrins are a large family of transmembrane receptors that connect cells to the extracellular matrix (ECM). Integrins function as heterodimers composed of $\alpha$ and $\beta$ subunits; there are $18 \alpha$ and $8 \beta$ integrins in mammals [2]. Different integrins bind to different ECM proteins [2]. For example, integrin $\alpha 5 \beta 1$ specifically binds to fibronectin, $\alpha 3 \beta 1$ to laminins, and $\alpha 1 \beta 1$ and $\alpha 2 \beta 1$ to collagens [3].

When cells migrate away, migrasomes adhere to the place where they are formed, which indicates that something holds the migrasome at its formation site. Mass spectrometry analysis revealed integrin $\alpha 5 \beta 1$ is enriched on migrasomes (unpublished data), suggesting this integrin is a possible candidate for the molecule that holds the migrasome on the ECM. To testify this hypothesis, we used MGC803 cells expressing TSPAN4-GFP that labels migrasomes, and stained the cells with antibodies against integrin $\alpha 5$ and $\beta 1$. We found that both integrin $\alpha 5$ and $\beta 1$ were enriched in migrasomes (Figure 1A). Moreover, using two antibodies which specifically recognize different epitopes of activated integrin $\beta 1$ (clone HUTS-4 and $12 \mathrm{G} 10$ ), we found migrasome-localized $\beta 1$ was in its activated ligand-binding state (Figure 1B), which indicates that the migrasomal integrin binds the ECM.

To study the migrasomal integrin in more detail, we transfected integrin $\alpha 5$-GFP into normal rat kidney cells expressing TSPAN4-mCherry (NRK-TSPAN4-mCherry). We found that integrin $\alpha 5$-GFP was highly enriched in migrasomes, whereas there was relatively less integrin $\alpha 5$-GFP on retraction fibers (Figure 1C). Time-lapse analysis revealed that during migrasome growth, small integrin-positive puncta first appeared on retraction fibers, and these integrin-positive puncta grew gradually and soon became TSPAN4-positive migrasomes (Figure 1D). Thus, the formation of integrin puncta appears to specify the site of migrasome formation.

Migrasomes are spherical structures, so if integrin molecules adhere migrasomes to the ECM, they must be located at the bottom of the migrasome. To test this hypothesis, we checked the 3D distribution of integrin $\alpha 5$ and TSPAN4 on migrasomes. We found that integrin $\alpha 5$ was mainly enriched on the bottom side of migrasomes while TSPAN4 was on the upper side (Figure 1E). Similarly, TIRF microscopy imaging revealed that endogenous integrin $\alpha 5$ and $\beta 1$ were enriched on the bottom of migrasomes (Supplementary information, Figure S1A and S1B). Taken together, these data on the spatio-temporal distribution pattern of integrin on migrasomes further support our hypothesis that integrin $\alpha 5 \beta 1$ adheres migrasomes to the ECM and may play important roles in migrasome formation.

Focal adhesions (FAs) are the sites that cells are linked to the ECM. Integrins are highly enriched in FAs [4]. We wondered whether the integrin-enriched regions on migrasomes are FAs. To test this idea, we co-transfected cells with various FA markers (paxillin, vinculin or zyxin) [5] and TSPAN4. We found that none of these FA markers was localized on migrasomes (Supplementary information, Figure S2A-S2C). Moreover, the average lifetime of migrasomes is about $200 \mathrm{~min}$, whereas the FAs are much more dynamic, with the lifetime of $<1 \mathrm{~h}$ (Supplementary information, Figure S2D). These data suggest that the integrin-enriched site on the bottom of the migrasome is not an FA. Interestingly, substrate-attached materials, which are cellular feet that remain on substrates after the treatment of adherent cells with EGTA, also contain integrins but not FA components [6].

Different integrins bind and adhere to different ECM proteins. We reasoned that migrasome formation may depend on the matching of specific integrin-ECM pairs. 

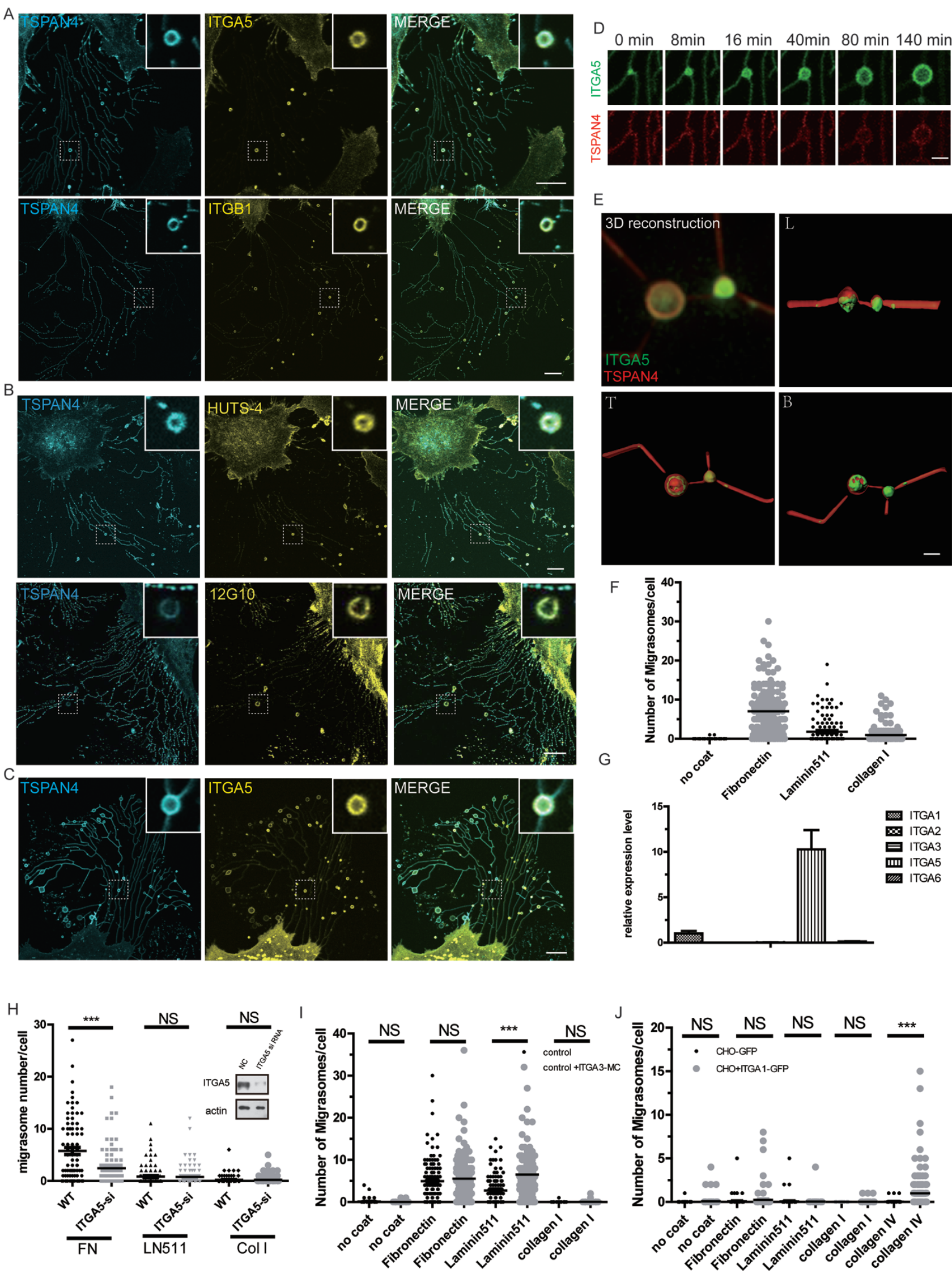
To test this hypothesis, we cultured TSPAN4-GFP-expressing NRK cells (NRK-TSPAN4-GFP) on different ECM proteins. We found that NRK-TSPAN4-GFP cells produced many more migrasomes on cover glasses coated with fibronectin than with laminin 511 or collagen I, whereas very few migrasomes formed on non-coated cover glasses (Figure 1F). Next, we checked the expression level of various integrins in NRK-TSPAN4-GFP cells, including integrin $\alpha 1$ and $\alpha 2$ that bind collagen I and IV, $\alpha 3$ and $\alpha 6$ that bind laminin 511, and $\alpha 5$ that binds fibronectin. We found that NRK-TSPAN4-GFP cells expressed much higher levels of $\alpha 5$ than other integrins (Figure 1G). Furthermore, knockdown of ITGA5 that encodes $\alpha 5$ impaired the formation of migrasomes on cells cultured on fibronectin, but not on other ECMs (Figure 1H). Thus, migrasome formation on NRKTSPAN4-GFP cells is likely dependent on integrin $\alpha 5$-fibronectin pairing.

In NRK-TSPAN4-GFP cells, the expression level of integrin $\alpha 3$ is very low. We overexpressed integrin a3-mCherry in NRK-TSPAN4-GFP cells, and found that it significantly enhanced migrasome formation on cover glasses coated with laminin 511, but not on cover glasses covered with other ECM proteins (Figure 1I). Due to the reason that GFP-tagged integrin $\alpha 1$ does not express well in NRK-TSPAN4-GFP cells, we used CHO cells to test whether integrin $\alpha 1$ can promote migrasome formation on paired ECM protein. Similarly, overexpression of GFP-tagged integrin $\alpha 1$ in $\mathrm{CHO}$ cells enhanced the formation of migrasomes on collagen IV, which has the highest binding affinity to integrin $\alpha 1$, but not on other ECM proteins (Figure 1J). Furthermore, overexpression of integrin $\alpha 1$ and $\alpha 3$ increased cell spreading and migration on their respective ECM partner protein (Supplementary information, Figure S3). This implies that integrin $\alpha 3$ and $\alpha 1$ may affect migrasome formation by regulating cell migration and adhesion. Similarly, overexpression of $\alpha 3$ and $\alpha 1$ in MGC803 cells, which have low endogenous levels of $\alpha 3$ and $\alpha 1$, also enhanced the formation of migrasomes on the respective ECM partner protein, but not on unmatched ECM proteins (Supplementary information, Figure S4). Based on these data, we conclude that the pairing of integrin with its specific ECM partner is a determinant for migrasome formation.

Migrasome formation depends on cell migration [1]. Formation of retraction fibers requires adherence of at least a portion of the retraction fiber to the cell surface to provide a tethering point, which enables the retraction fiber to be pulled from the trailing end of the cell as the cell migrates. The fact that integrins are enriched on the bottom of migrasomes, and that migrasomes are largely stationary while retraction fibers are often swaying around (Supplementary information, movie S1) indicates that the migrasome is the point of adhesion which tethers the retraction fibers to the ECM. Thus, integrins may play dual roles in migrasome formation: the integrins on the cell body enable the cell to migrate, whereas the integrin on the migrasome provides the adhesion for retraction fiber tethering.

In this report, we found that activated integrin is highly enriched on the bottom of migrasomes, and we further demonstrated that the expression level of integrin affects migrasome formation. Finally, we showed that the correct pairing of integrin with its specific ECM partner protein is a determinant for migrasome formation.

There are $18 \alpha$ and $8 \beta$ integrins in mammals and their expression is subject to various regulatory mechanisms. Different integrins bind different ECM proteins [2], and each ECM protein has a specific spatial and temporal distribution pattern in a given organism. Thus, our finding that pairing of integrins with their specific ECM partner proteins is a determinant for migrasome formation may

Figure 1 (A) Immunofluorescence (IF) staining of integrin $\alpha 5$ (ITGA5) and $\beta 1$ (ITGB1) in MGC803 cell line overexpressing TSPAN4-GFP. Scale bar, $10 \mu \mathrm{m}$. (B) IF staining of active integrin $\beta 1$ (HUTS-4 or 12G10) in TSPAN4-GFP-overexpressing MGC803 cells. Scale bar, $10 \mu \mathrm{m}$. (C) Live-cell images of NRK cells transfected with TSPAN4-GFP and integrin $\alpha 5-\mathrm{mCherry}$. Scale bar, $10 \mu \mathrm{m}$. (D) NRK cells were co-transfected with TSPAN4-mCherry and integrin $\alpha 5-G F P$, and time-lapse images were taken every $8 \mathrm{~min}$. Scale bar, $1 \mu \mathrm{m}$. (E) 3D reconstitution of TSPAN4-mCherry and integrin $\alpha 5-G F P$ on migrasomes and retraction fibers. T, top view; L, lateral view; B, bottom view. Scale bar, $2 \mu \mathrm{m}$. (F) TSPAN4-GFP-expressing NRK cells were cultured on different ECM proteins (fibronectin, laminin 511 and collagen I), and the number of migrasomes formed on each was counted. (G) The expression level of different integrin $\alpha$ subunits in TSPAN4-GFP-expressing NRK cells was analyzed by qPCR. (H) TSPAN4-GFP-expressing NRK cells were transfected with control or ITGA5-targeting siRNA for more than 24 $\mathrm{h}$, then plated on the FN-, LN511- or Col l-coated chamber overnight, and confocal pictures regarding migrasome number for each cell were taken. The western blotting analysis (the right panel) showed the knocking down efficiency. (I) NRK cells expressing TSPAN4-GFP alone (control) or TSPAN4-GFP with mCherry-tagged integrin $\alpha 3$ were cultured on different ECM proteins and the number of migrasomes per cell was counted. Data were analyzed with two-tailed $t$-tests (GraphPad Prism 5); ${ }^{* * *} P<0.001$. (J) CHO cells were transfected with pEGFP-N1 (control) or integrin $\alpha 1-\mathrm{GFP}$ and seeded into chambers coated with different ECM proteins, and the number of migrasomes per cell was counted. Data were analyzed with two-tailed $t$-tests (GraphPad Prism 5); ${ }^{* * *} P<0.001$. 
emerge as an important principle for determining where and when migrasomes can be generated in vivo.

So far, the detection of migrasomes has depended on TSPAN4, a marker we identified in our original paper. In this regard, integrins may serve as more specific markers for migrasomes (Figure 1A-1C and Supplementary information, movie S2). While both TSPAN4 and integrins are highly enriched on migrasomes, unlike TSPAN4 that is also abundant on retraction fibers, integrins are only present at very low levels on retraction fibers. Excellent integrin antibodies are available commercially, and thus they may emerge as important tools for studying migrasomes in vivo.

\section{Acknowledgments}

We are grateful to Nikon Instruments (Shanghai) and the Tsinghua Cell Biology Core Facility for providing technical support. This research was supported by Ministry of Science and Technology of the People's Republic of China (2017YFA0503404 and 2016YFA0500202), the National Natural Science Foundation of China (31430053 and 31321003), Natural Science Foundation of China international cooperation and exchange program (31561143002), and the Independent Research of Tsinghua University (20161080135).

Danni $\mathrm{Wu}^{1}$, Yue $\mathrm{Xu}^{2}$, Tianlun Ding ${ }^{1}$, Yan $\mathrm{Zu}^{2}$, Chun Yang ${ }^{2}$, Li Yu ${ }^{1}$

${ }^{1}$ State Key Laboratory of Biomembrane and Membrane Biotech- nology, Tsinghua University-Peking University Joint Center for Life Sciences, School of Life Science, Beijing 100084, China, ${ }^{2} I n-$ stitute of Biomechanics and Medical Engineering, School of Aerospace, Tsinghua University, Beijing 100084, China.

Correspondence: $\mathrm{Li} \mathrm{Yu} u^{\mathrm{a}}$, Chun Yang ${ }^{\mathrm{b}}$

${ }^{a}$ E-mail: liyulab@mail.tsinghua.edu.cn

bE-mail: yangchun@mail.tsinghua.edu.cn

\section{References}

1 Ma L, Li Y, Peng J, et al. Cell Res 2015; 25:24-38.

2 Zaidel-Bar R. Nat Cell Biol 2013; 15: 575-577.

3 Humphries JD, Byron A, Humphries MJ. J Cell Sci 2006; 119: 39013903.

4 Wehrle-Haller B. Curr Opin Cell Biol 2012; 24: 116-124.

5 Zaidel-Bar R, Cohen M, Addadi L, et al. Biochem Soc Trans 2004; 32:416-420.

6 Yamada M, Mugnai G, Serada S, et al. Cell Adh Migr 2013; 7: $304-$ 314.

(Supplementary information is linked to the online version of the paper on the Cell Research website)

\begin{abstract}
(c) (i)
This work is licensed under a Creative Commons Attribution 4.0 Unported License. The images or other third party material in this article are included in the article's Creative Commons license, unless indicated otherwise in the credit line; if the material is not included under the Creative Commons license, users will need to obtain permission from the license holder to reproduce the material. To view a copy of this license, visit http:// creativecommons.org/licenses/by/4.0/
\end{abstract}

(C) The Author(s) 2017 